\title{
A matching of soccer players for testing the association between skin tone and the proportion of red cards received
}

\begin{abstract}
I investigate the association between the skin tone of soccer players and the number of red cards they receive from a referee. This is done by matching players with a dark skin tone to players with a light skin tone, based on physically similarity of the players and the positions of the players in the field. No support was found for the presence of a positive association.
\end{abstract}

Key words: crowdsourcing; matching; skin-tone bias

\section{Introduction}

In the game of soccer, two teams of each eleven players compete in trying to score more goals than the opponent. In the game, players are allowed to make contact with other players, but a referee is presents at each game to judge whether the contact is within the limits set by the rules of the game, or whether a foul is committed. In case of a foul, the team against which player the foul was committed receives a free kick, and the referee may give either no card, a yellow card, or a red card to the player committing the foul. A player receiving a second yellow card during the game also gets a red card, and if a player receives a red card, that player has to leave the field, putting the team at a disadvantage. Other instances in which a player may receive a card include behaviours of bad sportsmanship.

Silberzahn et al. (2015) present a study by 29 teams of researchers, all addressing the question of whether referees are more likely to give red cards to dark skin toned players than light skin toned players, and all using the same data set. The study shows that the teams of researches vary widely in their choices of analyses and obtain varying results. For example, each team used a unique combination of covariates, with estimated effect sizes ranged from 0.89 to 2.93 (in terms of odds ratios). Lacking an experimental design, where one has some control over the confounding factors that otherwise obscure the results, the statistical adjustment resulting from the inclusion of covariates, is a way of accounting for pre-existing differences (i.e., confounding factors). However, the appropriateness of the inclusions of such covariates is contingent on the choice of model used for the analysis.

In this paper, the same data set is used as in Silberzahn et al. (2015), with the aim of addressing the same question, but without the use of covariates. Instead, a matching of players is considered, which results in a reduction of the complexity of the data structure to the analysis of a 2 by 2 table. 


\section{Method}

The data (Silberzahn et al., 2014) were obtained from Open Science Framework website (https://osf.io/gvm2z), and contains 146028 dyads of male players from the top league competitions of England, Germany, France, and Spain, and the referees encountered during the 2012-2013 competition. Each of these dyads contains the information about the club, league, age, height, weight, and position on the field of the player, along with the number of games that the player encountered the particular referee and the number of cards received from that referee. In addition, two independent ratings of the players tone of skin were available for each player. A perfect inter-rater agreement was obtained by joining the first two and last two points of the initial 5-point ratings of skin tones, and dropping from the analyses all players that obtained a mid-point rating from any of the two raters. Further removing any dyad containing missing observations on any of the above outcomes resulted in a total of 99481 dyads with 1212 players that were considered for matching, of which 199 were rated as having a dark-toned skin.

For the matching of players, the aim was to match a player of dark skin tone to a player of light skin tone, such that, 1) the two players each were paired with at least one and the same referee, 2) they played in the same league on one and the same position on the field, 3) and the two players were (apart from skin tone) physically similar. In order to assess the physical similarity of any two players that meet the first two requirements, the Mahalanobis distance between any of the potential matches was obtained using the correlations between age, height, and weight within each league. Only players with a Mahalanobis distance smaller than 3 were considered eligible matches (e.g., a players in Germany of 32 years, $191 \mathrm{~cm}$ tall, and weighing $81 \mathrm{~kg}$, could be matched to a player of 32 years, $177 \mathrm{~cm}$ tall, and weighing $76 \mathrm{~kg}$, but the same player could not be matched to a player of 23 years old, $182 \mathrm{~cm}$ tall, and weighing $76 \mathrm{~kg}$ ). In each league the number of dark skin-toned players were the minority. So, to maintain a large number of matches, the (heuristic) choice was made to iteratively match the dark skin-toned player with the fewest eligible matches to the light skin-toned player with the smallest Mahalanobis distance. This approach continued for the remaining players until no more eligible matches remained.

Across the four leagues, a total of 195 matches were obtained. Each of these matches were linked to one or more of the same referees, where I calculated for each player the proportion of red cards received across all the games by these referees. If the matching of players was successful, the skin tone is assumed to explain the association that may exist between the two players receiving a red card. As an indication of the success of the matching, the matched pairs, on average, had a Mahalanobis distance of .910 (corresponding to an average absolute difference of 2.256 years of age, $3.077 \mathrm{~cm}$ in height, and $3.087 \mathrm{~kg}$ in weight, with the players rated as dark-toned being on average eight months younger, less than $1 \mathrm{~cm}$ taller, and weighing less than 4 grams more than the light-toned players). Further, a multivariate analysis of variance showed no average difference between the matched players and unmatched players on age, 
height, and weight: $F(3,1208)=2.1382, p=.094$, suggesting the sample of matched players to be physically representative.

Given a successful matching, the probability of both players receiving a red card becomes the product of the proportion of red cards received by each player, and four proportions are obtained for each combination of cards received by the players. By summing up these proportions across the 195 matched pairs a 2 by 2 table of expected frequencies is obtained for testing the association between skin tone and receiving a red card $^{1}$.

The 2 by 2 table of the number of red cards received by the matched players was assumed to follow a multinomial kernel for the four expected frequencies, consisting of four probability parameters for each of the cells of the table. In line with the initial research question proposed by Silberzahn et al. (2015), two competing hypotheses about these parameters were considered: 1) the hypothesis of row-column independence and 2) the hypothesis of a positive association. The one-sided Bayes factor for testing row-column independence against a positive association is obtained in the Appendix. A Bayes factor smaller than unity can be interpret as evidence in favour of a positive association, brought about by the data (e.g., Wagenmakers, 2007).

\section{Results}

Table 1 shows the estimated frequencies of red cards for the matched pairs of players. The odds ratio for Table 1 is 1.542 , and thus in line with the range obtained by Silberzahn et al. (2015). For these observations, the one-sided Bayes factor was obtained to be 15.786 in favour of the row-column independence hypothesis (i.e., strong support) over the hypothesis of a positive association (see Appendix).

Table 1 Estimated Frequencies for the Matched Players

\begin{tabular}{|c|c|c|c|}
\hline \multirow{2}{*}{\multicolumn{2}{|c|}{ Red card }} & \multicolumn{2}{|c|}{$\begin{array}{l}\text { Light skin- } \\
\text { toned player }\end{array}$} \\
\hline & & No & Yes \\
\hline skin- & No & 191.673 & 1.487 \\
\hline toned player & Yes & 1.818 & .022 \\
\hline
\end{tabular}

\section{Discussion}

Ideally, if a player makes a foul that is judged by a randomly selected referee, one would also observe an exact replication of the game up to the point that the foul was committed, again judged by the same referee, but now with the player

\footnotetext{
${ }^{1}$ Note that, for each matched pair of players, one could randomly select a single game for each player and obtain one observation of the combination of cards received by both players. The expected frequencies across the 195 matched pairs is then the expectation across such randomly chosen games.
} 
having a different skin tone. Such an ideal observations would allow for a wellcontrolled assessment of the association that may exist between the skin tone of the player and the card received for making the foul. In this study, the next best thing was considered for making inferences about the association, by matching players with different skin tone. The goal was to illustrate the advantage that matching has over the many choices one would have in modeling the complex structures in the data. In essence, matching is like implementing a posteriori a design to the data.

\section{References}

Cook, J. D. (2005). Exact calculation of beta inequalities. MDACC technical report UTMDABTR-005-05.

Cook, J. D. \& Nadarajah, S. (2006). Stochastic inequality probabilities for adaptively randomized clinical trails. Biometrical Journal, 48, 356-365.

Gunel, E., \& Dickey, J. (1974). Bayes factors for independence in contingency tables. Biometrika, 61, 545-557.

Morey, R. D. \& Wagenmakers, E., -J. (2014). Simple relation between Bayesian order-restricted and point-null hypothesis tests. Statistics $\&$ Probability Letters, 92, 121-124.

Silberzahn, R., Uhlmann, E. L., Martin, D. P., Nosek, B. A. (2014, November 19). CrowdstormingDataJuly1st.csv (raw data). Retrieved from https://osf.io/47tnc/

Silberzahn, R., Uhlmann, E. L., Martin, D. P., Pasquale, Aust, F., Awtrey, E. C., ... Nosek, B. A. (2015, August 20). Many analysts, one dataset: Making transparent how variations in analytical choices affect results. Retrieved from https://osf.io/j5v8f/

Wagenmakers, E. -J. (2007). A practical solution to the pervasive problems of $p$ values. Psychonomic Bulletin \& Review, 14, 779-804.

\section{Appendix}

Let $\Theta_{i j}(1 \leq i, j \leq 2)$ denote the random variable for the probability parameter of the multinomial distribution on the 2 by 2 table, where $\sum_{i, j} \theta_{i j}=1$, and $n . .=195$ (i.e., $n_{11}=191.673, n_{12}=1.487, n_{21}=1.818$, and $n_{22}=.022$ ). The hypothesis 1 of row-column independence states that $\theta_{i j}=\theta_{i \cdot} \cdot \theta_{\cdot j}$, where we assume the prior of $\left(\Theta_{i j}\right)$ to be Dirichlet $(1,1,1,1)$, resulting in the posterior $\operatorname{Dirichlet}\left(n_{11}+1, n_{12}+1, n_{21}+1, n_{22}+1\right)$. Gunel and Dickey (1974) provide an expression for the Bayes factor $L_{01}$ for testing hypothesis 1 against the hypothesis that does not impose contains on the parameters (hypothesis 0), given 
a multinomial sampling design. Applying Equation 4.4 by Gunel and Dickey yields $L_{01}^{-1}=30.535$.

A one-sided Bayes factor for testing hypothesis 1 against the hypothesis of a positive association (hypothesis 2) is obtained as $L_{12}^{-1}=x L_{01} / y$ (cf. Morey \& Wagenmakers, 2014), where $1 / x=1 / 2$ is the prior probability of a positive association, and $1 / y$ is the posterior probability of a positive association. As the positive association $\theta_{11} / \theta_{21}>\theta_{12} / \theta_{22}$ is an expression of odds of gamma variables, the inequality corresponds to an inequality between beta variables, for which Cook (2005; Cook \& Nadarajah, 2006) provides an exact expression. First, let $a=n_{11}-192, b=n_{21}-1, c=n_{12}-1$, and $d=n_{22}$, and let $g(a, b, c, d)=P\left(\theta_{11} / \theta_{21}>\theta_{12} / \theta_{22}\right)$. With $a+b+c+d=1$ an exact expression for $g(a, b, c, d)$ is given by Cook \& Nadarajah (2006, Equation 4). By applying the recurrence relations therein (Cook \& Nadarajah, 2006, p. 359) until, $a=n_{11}+1$, $b=n_{21}+1, c=n_{12}+1$, and $d=n_{22}+1$, we get the posterior probability $1 / y=.967$. Consequently, $L_{12}=15.786$ (an exact solution). 